\title{
ESTRATEGIAS DE DECISIÓN DE UN JURADO BAJO CONDICIONES DE SIMULACIÓN
}

\author{
E. SAINZ \\ Universidad de Granada \\ J. SAINZ \\ Universidad Complutense de Madrid
}

\section{Resumen}

El objeto de esta investigación es analizar el pro. ceso de decisión de un jurado bajo condiciones de simulación. A traves de dos experimentos en que se manipula la forma de presentación de la intormacion, se estudian los efectos de la regla de decisión y de las instrucciones en el veredicto y los efectos de la deliberación. Se defiende la hipótesis de que la regla de decisión y las ins. trucciones tienen, respectivamente, efectos informativos y normativos sobre el veredicto, y que la deliberación tiene efectos informativos. Estos efectos, sin embargo, sólo resultan parcialmente evidentes en el contexto de una estra. tegia de juicio compensatoria que supone el pro. cesamiento analitico de la evidencia aportada en el juicio. Los resultados muestran que los sujetos cambian de estrategia cuando cambia la forma de presentación y el tipo de respuesta solicitada que es holistica, de elección y no compensatoria en la presentación simuitanea y analítica. de juicio y compensatoria en la presentación se. cuencial. Sólo cuando la deliberación supone un cambio de estrategia tiene un efecto sobre el ve. redicto que es mas favorable que lo que lo era inicialmente para el acusado. Los resultados parecen adecuarse a un modelo de acumulación de evidencia que afecta a la evaluación de los items incriminatorios del caso.

\section{Abstract}

The focus of the present research is to simulate and analyze the jurors' decision process. The effects of the decision rule and the instructions on the veredict were studied, using experiments in which the presentation of the information was manipulated. The effect of the deliberation process was also studied. It is postulated that decision rule and instructions have informative and normative effects, respectively, on the veredict, and that deliberation has informative effects. $\mathrm{Ne}$. vertheless, these effects are only partially evident in the context of a compensatory judgment strategy. which assumes the analytic processing of the evidence presented in the trial. Results showed that subjects' strategies changed when the presentation of the information changed, and when the response mode demanded was holistic, forced a choice, and was not compensatory in a simultaneous presentation, and analytic judgmental and compensatory in a sequential presentation. Only when the deliberation brings out a change in strategy it does have an effect on the veredict, which is to make it more favora. ble than what it initially was for the defendant. The results support a model of cumulative evidence that affects the evaluation of the incriminating items in the case.

\section{Introducción}

La cuestión de la discrecionalidad que puede permitirse a los distintos componentes del sistema judicial a la hora de tomar sus decisiones es una de las cuestiones críticas que se plantean ante cualquier reforma que se pretenda del mismo. La implantación del jurado en nuestro ámbito se discute, a menudo, desde los sectores profesionales del sistema judicial, partiendo del supuesto de que el lego en derecho no es el mejor garante de los derechos del ciudadano, acusado de un delito, en un estado de- mocrático. Sin embargo, el problema del grado de discreción que puede permitirse a un jurado no es exclusivo de él, aunque en apariencia pueda parecer más dramático por el hecho de que no pueda imponérsele un rigor y una disciplina que si parecen exigibles, en cambio, a los funcionarios. Para cumplir sus fines el Estado debe velar por el control de la discreción extrajurídica de los funcionarios, máxime allí donde se da un tipo de organización profesional más que burocrática (Feeley, 1983), donde la racionalidad se atribuye a los individuos y a su moral particular más que residir en el conjunto de la orga- 
nización, en el establecimiento de controles internos por parte de ésta mediante la división de funciones, la organización jerárquica y la supervisión. Que prevalezca un tipo de organización burocrática frente a una de carácter profesional, incomprensible para los ciudadanos, depende de la legitimación política que introduce el jurado en el sistema judicial, en tanto representa el origen de las leyes (Scheflin y VariKike, 1980), y refrenda o dëtermina su interpretación.

En este contexto, el análisis de las decisiones que toman los distintos agentes del sistema judicial es una cuestión crítica para su justificación racional. El objeto de esta investigación es analizar el proceso de decisión de un jurado, bajo condiciones de simulación, y más específicamente las "condiciones de existencias (cfr. Wallsten y Budescu, 1983), bajo las que los miembros de un jurado emiten sus veredictos, condiciones que afectan a la calidad de sus decisiones. El análisis de estas condiciones permite determinar en qué términos puede generalizarse este tipo de investigaciones de laboratorio a la vida real (Christensen-Szalanski, 1986), y de qué modo debería regularse la implantación del jurado.

El control de la discreción de un jurado, donde se cuenta con tradición suficiente, se ha hecho estableciendo leyes, en primer término, respecto de la selección de sus miembros, de su tamaño y composición, $y$, en segundo término, mediante normas respecto de las instrucciones y la regla de decisión que ha de regir su veredicto tras la deliberación, o que rigen su intervención durante el juicio. Respecto de las instrucciones, se han considerado dos clases: a) las que se refieren a la presunción de inocencia, que exige del jurado que se atenga a la evidencia para la emisión de su veredicto; y $b$ ) las que se refieren a los rasgos que caracterizan una cierta categoria o clase de veredictos: relativas a los roles de los participantes, a los estados que afectan a los participantes y acciones que llevan a cabo, y a las circunstancias que les afectan. Respecto de las reglas, se han considerado, asimismo, dos clases, al menos: a) una regla de unanimidad que requiere el consenso de todos los miembros de un jurado en tomo $a$ un veredicto; y b) una regla de mayoría simple 0 absoluta según las distintas altemativas de veredicto y la discrecionalidad permitida al jurado.

Kerr, Atkin, Stasser, Meek, Holt y Davis (1976) sometieron a prueba la hipótesis de que si se proporcionaban a un jurado instrucciones muy estrictas deberia obtenerse una tasa de veredictos de culpabilidad menor que si se le proporcionaban instrucciones no estrictas. Analizando los resultados de una investigación en la que se estudiaba el proceso de toma de decisiones de un jurado bajo condiciones de simulación, encontraron un efecto de las instrucciones que confirmaba la hipótesis. Davis, Kerr, Atkin, Holt y Meek (1975) en una investigación anterior habian sometido a prueba $r$ hipótesis de que una regla de decisión más estricta -la regla de unanimidad- debería dar lugar a una tasa de veredictos de culpabilidad menor que una regla de decisión menos estricta - la regla de mayoria de dos tercios-. Analizando los resultados obtenidos, no en- contraron ningún efecto significativo asociado a la regla de decisión utilizada.

La emisión de un veredicto, por parte de un jurado, es el resultado de la información que se aporta durante el juicio, de la forma en que esta información se procesa (Pennington y Hastie, 1986) y de la forma en que se evalúa de acuerdo con la influencia que el propio jurado ejerce sobre cada uno de sus miembros (Tanford y Penrod, 1986). Sin duda, el análisis de una decisión compleja como ésta requiere un análisis más detallado de cómo la deliberación del jurado afecta al análisis y evaluación de la evidencia y a la emisión de un juicio atendiendo a factores de carácter normativo. Billings y Scherer (1988) han distinguido, sin embargo, entre juicio y elección. En tanto el juicio requiere una evaluación explícita de cada una de las alternativas que se consideran, la elección supone la adopción o selección de una alternativa frente a otras que se rechazan. Esta distinción es importante, ya que la respuesta que se requiere en una tarea puede tener un efecto sobre los procesos que, en última instancia, conducen a la adopción de una decisión. De acuerdo con Billings y Scherer (1988), el modo de respuesta puede ser una variable crítica en la emisión de un veredicto determinando la forma en que procede el jurado a la hora de tomar una decisión.

El primer objetivo de esta investigación es contrastar las hipótesis de Kerr, Atkin, Stasser, Meek, Holt y Davis (1976) en relación con la regla de decisión, y de Davis, Kerr, Atkin, Holt y Meek (1975) en relación con las instrucciones, manipulando las condiciones en que un jurado procesa la información proporcionada del caso o casos que juzga. Manipulando el tipo de respuesta que se solicita es posible confirmar las tesis de Kerr y sus colaboradores $(1975,1976)$ en relación con los efectos de la regla de decisión y de las instrucciones sobre el proceso de toma de decisiones. El tipo de respuesta tiene un efecto sobre el uso de estrategias de decisión compensatorias o no compensatorias en función de las que el sujeto decide evaluar, respectivamente, la información que se aporta para cada una de las alternativas o éliminar aquella información que no conduce a una cierta decisión. Nuestra tesis es que el sujeto hará una evaluación holistica de la información cuando ésta se presente simultáneamente y una evaluación analítica cuando se presente secuencialmente (cfr. Payne, 1982). En el primer caso, el juicio tendrá lugar, propiamente, cuando el jurado evalúe la información durante la deliberación (cfr. Bettman, 1982; Eihorn, Kleinmuntz, y. Kleinmuntz, 1979), en tanto en el segundo cada sujeto tomará una decisión a medida que vaya evaluando la evidencia aportada, es decir, antes de la deliberación. En otros términos, un sujeto evalúa la información de acuerdo con una estrategia inicialmente no compensatoria cuando la evaluación es holística y de acuerdo con una estrategia inicialmente compensatoria cuando la evaluación es analítica (cfr. Tversky, 1977).

El segundo objetivo de esta investigación se refiere a la clase de influencia que el jurado ejerce como 
grupo sobre cada uno de sus miembros. En repetidas ocasiones se ha señalado que los sujetos adoptan, a menudo, la decisión del grupo sólo por lo que se refiere a la norma, pero que rara vez modifican sus puntos de vista sobre el valor que hay que dar a la información presentada (cfr. Tanford y Penrod, 1986). A pesar de la importancia de esta tesis, no se han realizado investigaciones experimentales que permitan distinguir la influencia normativa $\theta$ informativa que un jurado ejerce sobre cada uno de sus miembros desde la perspectiva de las estrategias que utilizan para evaluar la evidencia: Si la deliberación del jurado tiene un efecto informativo y no exclusivamente normativo, una estrategia de procesamiento de información más analítica puede reducir 0 , incluso, eliminar, el efecto de la deliberación. La influencia del jurado se reduciría al criterio adoptado por cada uno de sus miembros para determinar si el acusado es o no efectivamente culpable. Nuestra predicción es que la deliberación tiene un efecto sobre el procesamiento analítico de la información y que el jurado como tal no afecta a la toma de una decisión sino a través del criterio de decisión a adoptar por cada uno de sus miembros. Las influencias informativa y normativa de un jurado sobre cada uno de sus miembros pueden ser, por tanto, independientes.

\section{EXPERIMENTO 1}

Un aspecto critico para el análisis del proceso de toma de decisiones por parte de un jurado es el que se refiere a la forma en que las instrucciones controlan su conducta. La selección de un tipo de instrucciones debe, por tanto, justificarse. El propósito de este primer experimento es instrumental; se trata de verificar si los dos tipos de instrucciones que se presentan, moderadas y fuertes, se distinguen entre si, respecto de la certeza que exigen en el veredicto.

\section{Método}

\section{Sujetos}

Diez sujetos, de edades comprendidas entre los 20 y 44 años, cinco hombres y cinco mujeres, estudiantes del Instituto de Criminología de la Universidad de Valencia, participaron voluntariamente, satisfaciendo un requisito opcional del curso.

\section{Diseño y procedimiento}

El objetivo de este primer experimento era comprobar si las instrucciones adoptadas como fuertes y moderadas por el investigador resultaban ser efectivamente distintas para los sujetos en una aplicación posterior. Los sujetos experimentales participaban, entonces, como jueces de las instrucciones, que evaluaban, cada una por separado, en un cuestionario compuesto de una serie de escalas relativas a los grados de certeza y confianza que exigen las instrucciones, y al grado en que son comprensibles. El cuestionario incluia, asimismo, con un fin descriptivo, el test del umbral de decisión de Nagel, Lamm y Neef (1981).

Se distribuian a los sujetos por separado y en un orden aleatorio dos tipos de instrucciones, fuertes y moderadas, solicitando que las leyeran con cuidado. Tras la lectura de cada instrucción se les pedia que rellenaran un cuestionario que incluia el test del umbral de decisión y las escalas ya mencionadas.

\section{Material de estimulo}

El material de estimulo consistía en dos tipos de instrucciones, fuertes y moderadas, respecto del grado de certeza que hay que alcanzar para declarar a alguien culpable (véase apéndice l). Estas instrucciones se elaboraron a partir de las utilizadas por Kerr, Atkin, Meek, Holt y Davis (1976), adaptándolas a las condiciones de una muestra española e igualando su relativa extensión (Sainz, 1988). A la presentación de cada uno de estos tipos de instrucción le seguía un cuestionario en que se presentaban una serie de escalas continuas de 0 a 100 en cada una de las cuales el sujeto habia de determinar su respuesta. El cuestionario contaba con una escala para determinar el umbral de decisión y escalas de certeza, confianza y comprensibilidad para juzgar cada tipo de instrucción.

\section{Resultados y discusión}

Las puntuaciones medias en la escala de certeza de las instrucciones fuertes $(72,55)$ y moderadas $(32,66)$ son significativamente distintas entre sí, $t(2,18)=3,02, p<0,01$. No resultan ser en cambio significativas las diferencias que se obtienen respectivamente, entre ambos tipos de instrucción, en las escalas de confianza $(62,77$ vs 47,88$)$ y comprensibilidad $(87,66$ vs 72,88$)$. La fiabilidad por el método de las dos mitades era de $r=0,89$. Sometiendo las puntuaciones directas, obtenidas en las siete escalas, a un análisis factorial de componentes principales, el 87 por 100 de la varianza puede explicarse en términos de tres factores; en el espacio factorial, el factor de coherencia, que carga positivamente en las escalas que califican las instrucciones fuertes y negativamente en las que califican las instrucciones moderadas, explica el 39,87 por 100 de la varian2a; el factor de prudencia, que carga positivamente en la escala del umbral y en la escala de comprensibilidad de las instrucciones fuertes, y negativamente en confianza y certeza de las instrucciones moderadas, explica el 36,24 por 100 de la varianza; $y$, por último, el factor de confianza, que carga positivamente en la escala de comprensión de las instrucciones moderadas y negativamente en la certeza que exigen las instrucciones fuertes y moderadas, explica el 23,89 por 100 , el resto de la varianza total. 
De acuerdo con nuestros resultados los sujetos distinguen entre ambos tipos de instrucción, es decir, en la certeza que se exige para declarar al acusado culpable. Ambos tipos de instrucción se comprenden en ol mismo grado y exigen la misma confianza. Los tres factóres que se identifican en el análisis factorial aportan una perspectiva acerca de las condiciones que respaldan la evaluación de estas instrucciones; estos factores parecen definir los componentes implicitos en el criterio de dudá razonable.

\section{EXPERIMENTO 2}

En este experimento, tratamos de contrastar las hipótesis de Kerr y colaboradores $(1975,1976)$ en relación con los efectos de la regla de decisión y las instrucciones en el proceso de toma de decisiones de un jurado cuando la información del caso se presenta simultáneamente. En este contexto, el sujeto debe proceder, de acuerdo con una estrategla de decisión no compensatoria, en términos de una elección, en la que evalúa de forma contrastiva, por relación al veredicto, la información aportada en el juicio. Es razonable, por tanto, esperar que el sujeto evalúe la información analíticamente durante la deliberación del jurado. La deliberación reducirá, por hipótesis, la tasa de veredictos de cúlpabilidad al requerir de los sujetos una evaluación más precisa de la evidencia disponible. La deliberación introduce cambios en el procesamiento de la información que afectan a la evaluación de la evidencia. En ausencia de un análisis exhaustivo de ésta, la influencia del jurado sobre cada uno de sus miembros no es sólo de carácter normativo sino también de carácter informativo. En tanto las instrucciones tienen un carácter estrictamente normativo, al afectar al criterio de presunción de inocencia, la regla de decisión ha de afectar presumiblemente al tratamiento que es preciso hacer de la información. El hecho de que Davis, Kerr, Atkin, Holt y Meek (1975) no encontraran ningún efecto de la regla de decisión puede deberse a que, en la deliberación, prime la influencia normativa del jurado sobre el procesamiento de información. El sujeto puede tener la expectativa de que un análisis más profundo de la información puede hacerle más consistente frente al grupo: De acuerdo con esta interpretación, la regla de decisión, si se conoce por anticipado, como en nuestro caso, puede tener un efecto mayor cuando el sujeto está procesando la información más que cuando la discute con otros durante la deliberación. Aunque esta última hipótesis no puede contrastarse en este experimento - dado que sólo se obtiene una tasa de veredictos predeliberatoria-, parece razonable esperar que la regla de decisión, como tal, no tenga ningún efecto, dado que el sujeto no evalúa analíticamente la información que se le presenta hasta la deliberación. Durante la deliberación, los efectos de la regla de decisión, por hipótesis de naturaleza informativa, se ven postergados ante los efectos de naturaleza normativa introducidos por el jurado.
Este resultado estanía de acuerdo con lo que hallaron Davis; Kerr, Atkin, Holt y Meek (1975) en su investigación experimental.

\section{Método}

\section{Sujetos}

Ciento ocho sujetos efectivos, de edades comprendidas entre los 19 y 36 años, estudiantes de las facultades de. Derecho $(n=36)$, Económicas $(n=36)$, y de la Escuela de Trabajo Social $(n=36)$ de la Universidad de Valencia participaron voluntariamente en este segundo experimento. Estos sujetos se distribuyeron al azar en 18 grupos de seis personas cada uno, viniendo ya formados a la clta experimental. La relación por sexos era de un 58 por 100 de mujeres y un 42 por 100 de hombres. Seis de estos grupos procedian de la sustitución de otros tantos que se descartaron por no acudir todos sus miembros (4) o por no cumplir con los requisitos de la tarea (2).

\section{Diseño}

Se empleó un diseño factorial completo 2 (reglas de decisión: mayońa simple o unanimidad) $\times 3$ (tipos de instrucción: débiles, moderadas y fuertes) $\times 4$ (casos de robo: Julio de Castro, Juan Aguilera, Guillermo Lizcano, y José Linos; véase apéndice II). Como variable dependiente se adoptó el veredicto predeliberatorio y el veredicto postdeliberatorio del sujeto, agrupado para los cuatro casos, y expresado como una proporción, es decir, dos medidas repetidas únicas de los juicios con que los sujetos calificaban la culpabilidad o no culpabilidad de los cuatro acusados. A fin de llevar a cabo un análisis descriptivo de las variables que afectan a esta decisión se tomaron medidas escalares adicionales relativas al umbral de decisión, la confianza en el veredicto escogido, la probabilidad de que el acusado fuera culpable y la importancia relativa de las pruebas de la acusación y de la defensa. Los sujetos se asignaron al azar a cada condición experimental con la limitación de que hubiera seis miembros en cada grupo y se completaran los dieciocho grupos previstos.

Los tres tipos de instrucción incluyen las instrucciones moderadas y fuertes que se distinguieron en el primer experimento, más una nueva condición que, por conveniencia, denominamos débiles. Sin embargo, este último tipo de instrucción carece por completo de este carácter. En efecto, no incluye ningún tipo de definición acerca del criterio de duda razonable, al contrario que las instrucciones moderadas y fuertes, y se constituye únicamente del cargo de la acusación. Los cuatro casos que se someten a juicio son adaptaciones de los utilizados por Kerr (1985b). respetando la estructura e importancia relativa de las pruebas favorables a la acusación y a la defensa. Todos estos casos son balanceados respecto de la tasa de veredictos favorables y no favorables a que dan lugar. 


\section{Material de estímulo}

El material de estímulo consistia en un test del umbral, único y general para todos los casos; una hoja descriptiva de la evidencia de cada caso, y dos cuadernillos de respuesta idénticos, uno predeliberatorio, y otro postdeliberatorio para cada caso. La información relativa a cada caso se presenta de una șola vez y de forma unitaria. La única diferencia entre ambos cuadernillos afecta al test de disposición inicial de Ostrom, Werner y Saks (1978) que sólo se incluye en el primer cuadernillo. Ambos cuadernillos incluyen, para cada caso, una pregunta que solicita la elección de un veredicto, una serie de escalas continuas de 0 a 100 que tratan de medir la confianza que se tiene en el veredicto escogido, la probabilidad subjetiva de que el acusado sea culpable, la fuerza estimada de las pruebas de la acusación y de la defensa, y dos últimas preguntas, abiertas, referentes a las pruebas favorables al veredicto escogido $y$ otras que seria preciso practicar para estar más seguro de este veredicto. Estas preguntas cumplian la función de provocar un procesamiento significativo y cuidadoso de la información y servían, por tanto, como control de la calidad del trabajo desarrollado por los sujetos.

El cuestionario predeliberatorio se rellenaba previo a la deliberación del grupo. El cuestionario postdeliberatorio tras la deliberación del mismo. Este último cuadernillo incluia una pregunta adicional acerca del veredicto grupal y el número de votos a favor y en contra de la decisión adoptada.

\section{Procedimiento}

Esta investigación se presentaba brevemente, en cada condición experimental, como un estudio del proceso de toma de decisiones de un jurado. Previa a la administración de la hoja descriptiva de cada caso y el cuestionario predeliberatorio, los sujetos rellenan, de forma individual, el test del umbral. Una vez rellenado el test del umbral y antes de rellenar el primer cuadernillo se les comunica, de acuerdo con el diseño experimental; una regla de decisión y un tipo de instrucción. Rellenado el primer cuadernillo, los seis miembros de cada grupo se reúnen a deliberar sobre el caso durante un tiempo mínimo de diez minutos por caso, autoadministrándose el tiempo. Los casos se presentan en un orden aleatorio, previamente determinado, para cada grupo. Tras la deliberación rellenan el cuestionario postdeliberatorio, concluyendo la tarea con el veredicto final del grupo.

\section{Resultados}

Una primera aproximación a nuestros resultados nos proporciona una descripción de las variables de umbral y predisposición que afectan al proceso de decisión de un miembro de un jurado. El umbral de decisión en el test de Nagel, Lamm y Neff (1981) alcanza, en nuestra muestra, una puntuación media de 57,20619 en el rango esperado. La disposición inicial on el test de Ostrom, Wemer y Saks (1978), que expresa la probabilidad subjetiva de que un acusado de un delito sea efectivamente culpable, pone al descubierto una tendencia constante de los sujetos a juzgar más probable la culpabilidad del acusado cuando aumenta la gravedad del delito de que es acusado (una escala que varía entre una puntuación media de 60,55 a una puntuación media de 72,31). Esta tendencia subjetiva refleja además la creencia de que el sistema judicial es más eficiente con los delitos más graves que con los menos graves. El sujeto tiende a confundir las preferencias en la selección de los casos de que se ocupa el sistema judicial con la eficiencia con que trabaja. Se tiende a pensar que un caso cuanto más grave va a contar con más y mejor evidencia.

Una escala de probabilidad subjetiva, incorporada al test de Ostrom, Werner y Saks (1978), que permite juzgar la probabilidad de que un acusado que no ha cometido de hecho el delito de que se le acusa sea declarado culpable, por parte del juez, refleja que los sujetos atribuyen al juez su mismo umbral de decisión, una puntuación media de 59,22. Las puntuaciones de confianza predeliberatorias, por una parte, y postdeliberatorias, por otra, son semejantes entre sí en todos los casos, pero se observa tras la deliberación grupal un aumento generalizado y significativo en esta escala. Asimismo, tras la deliberación se observa una pérdida de significación de las pruebas de la acusación $(50,86$ vs 46,94$)$ a favor de las pruebas de la defensa $(39,79$ vs 42,83$)$ y un leve aumento medio, aunque significativo, de las estimaciones de probabilidad de no-culpabilidad $(43,05$ vs 45,77 ), más favorables al acusado. La descripción de estos efectos puede ser contrastada en el análisis de varianza.

Los sujetos producen tasas de veredictos de culpabilidad en general equilibradas, del orden de un 31,3 por 100 frente a un 23 por 100 en la Escuela de Trabajo Social, de un 55,2 por 100 frente al 48,3 por 100 en la Facultad de Económicas, y de un 52,2 por 100 a un 48,2 por 100 en la Facultad de Derecho, antes y después de la deliberación respectivamente. Observemos que todas las tasas de veredictos resultan ser menores tras la deliberación, aun cuando son puntuaciones individuales y se han tratado de forma independiente, una razón de sus bajas, aunque significativas, diferencias mutuas.

Las correlaciones que se obtienen entre las escalas que evalúan la relevancia de las pruebas de la acusación y la probabilidad subjetiva de culpabilidad por una parte, y la relevancia de las pruebas de la defensa y la probabilidad subjetiva de no-culpabilidad por otra, son positivas y significativas $(r=0,59$ $y r=0,59, p<0,01$, antes y después de la deliberación, respectivamente). A su vez, las correlaciones que se obtienen entre estas escalas y sus condiciones opuestas son negativas $y$ significativas $(r=-0,46$ y $r=-0,50, p<0,01$, antes y después de la deliberación, respectivamente).

A fin de ejecutar los sucesivos análisis sobre distribuciones normalizadas de los datos originales, se 
calcularon los antilogantmos de las puntuaciones medias obtenidas en las distintas escalas (escalas de umbral de decisión, conflanza, probabilidad de culpabilidad, fuerza de la evidencia a favor de la acusación y fuerza de la evidencia a favor de la defensa) para los cuatro casos y de la proporción de veredictos de no-culpabilidad sobre los cuatro casos.

Sometiendo las puntuaciones escalares predeliberatorias obtenidas, transformadas de esta forma en una escala loganítmica, a un análisis factorial de componentes principales, el 55,82 por 100 de la varianza en el espacio de los datos puede explicarse en términos de dos factores; en el espacio factorial, el factor de culpabllidad, en el que ponderan positivamente las escalas que califican la fuerza de las pruebas de la acusación, la probabilidad de que el acusado sea culpable y la tasa de veredictos, y negativamente la que califica el umbral de decisión, explica el 66,53 por 100 de la varianza; el segundo factor de no-culpabilidad, en el que ponderan positivamente las escalas que califican la fuerza de las pruebas de la defensa, y negativamente la confianza en la decisión adoptada, la probabilidad de que el acusado sea culpable y la tasa de veredictos, explica el 33,47 por 100 de la varianza (cfr. cuadro 1).

Sometiendo a análisis factorial las puntaciones escalares postdeliberatorias se obtiene una pauta de resultados y una configuración factorial relativamente distinta y significativa. Do nuevo, dos factores permiten explicar el 57,89 por 100 de la varianza en el espacio de los datos; el primer factor de culpabilidad, en el que ponderan positivamente las escalas de probabilidad de culpa, fuerza de las pruebas de la acusación, y tasa de veredictos y negativamente la fuerza de las pruebas de la defensa, explica el 67,94 por 100 de la varianza en el espacio factorial; el segundo factor de no-culpabilidad, en el que pondera positivamente la escala en la que se juzga el umbral de decisión y negativamente la fuerza de las pruebas de la acusación, la confianza y la tasa de veredictos, explica el 32,06 por 100 de la vanianza. Los cambios que se observan entre los juicios que los sujetos emiten antes y después de la deliberación nos proporcionan una descripción del efecto que la deliberación tiene sobre el proceso de decisión (cf. cuadro 1).
Un análisis de regresión múltiple, llevado a cabo para detectar el mejor conjunto de predictores del veredicto predeliberatorio, muestra que las variables umbral de decisión, probabilidad de culpabilidad, pruebas a favor de la acusación y pruebas a favor de la defensa explican, conjuntamente, el 42,06 por 100 de la varianza, $F(4 ; 103)=18,70, p<0,001$, Un modelo que incluye el segundo mejor conjunto de predictores, definido por la variable probabilidad de cuipa, y las variables pruebas a favor de la acusación y pruebas a favor de la defensa, permite explicar el 37,82 por 100 de la varianza $F(3,104)=21,09$, $p<0,001$. Una reducción no significativa de poco más de un 4 por 100 . Un análisis del mismo carácter, con objeto de predecir el veredicto postdeliberatorio, muestra que las variables del conjunto definido por las variables umbral de decisión, y las variables pruebas a favor de la acusación y de la defensa, explican el 42,85 por 100 de la varianza, $F(3,104)=25,99, p<0,001$. Bastarían, sin embargo, las variables pruebas a favor de la acusación y pruebas a favor de la defensa para predecir el 39,53 por 100 de la varianza, $F(2,105)=34,31$, $p<0,001$, con una reducción de poco más del 3 por 100 , no significativa. Atendiendo al mejor conjunto incluiriamos todas las variables que entran en el análisis con un aumento apenas significativo de un 3 por 100 adicional de explicación de la varianza $(F(5,102)=17,30, p<0,001$ con el 45,88 por 100 de la varianza explicada).

Los resultados individuales obtenidos en tasa de veredictos antes y después de la deliberación, se sometieron, una vez transformados en una escala logarítmica, a un análisis de varianza mixto con casillas de igual tamaño. Las puntuaciones directas predeliberatorias y postdeliberatorias se transformaron, agrupando los veredictos de los cuatro casos y estableciendo el porcentajo de veredictos de noculpabilidad proporcionados por cada sujeto, en logaritmos neperianos. Los valores absolutos de estas puntuaciones constituyen las dos medidas repetidas de la variable dependiente. Como se trataba de normalizar la distribución de las puntuaciones obtenidas, sustituimos las puntuaciones de $1,00 \mathrm{y}$ de 0,00 en tasa de veredictos por 0,99 y 0,01 respectivamente. La razón es que, por hipótesis, con el suficiente número de sujetos y de casos resultan margi-

\section{CUADRO 1}

Pautas y cargas factoriales rotadas predeliberatorias y postdeliberatorias en los análisis factoriales sobre las puntuaciones escalares y tasas de veredicto del segundo experimento

\begin{tabular}{|c|c|c|c|c|c|c|c|}
\hline \multirow{2}{*}{ Pauta } & \multirow{2}{*}{ Var. } & \multicolumn{2}{|c|}{ Predellberatorio } & \multirow{2}{*}{ Pauta } & \multirow{2}{*}{ Var. } & \multicolumn{2}{|c|}{ Postdeliberatorio } \\
\hline & & Factor 1 & Factor 2 & & & Factor 1 & Factor 2 \\
\hline $\begin{array}{l}\text { Acusación } \\
\text { Veredicto } \\
\text { Umbral } \\
\text { Defensa } \\
\text { Confianza } \\
\text { Prob. Culp }\end{array}$ & $\begin{array}{l}5 \\
2 \\
1 \\
6 \\
3 \\
4\end{array}$ & $\begin{array}{r}0,826 \\
0,707 \\
-0,522 \\
0,113 \\
0,063 \\
0,513\end{array}$ & $\begin{array}{r}-0,028 \\
-0,458 \\
-0,077 \\
0,840 \\
-0,591 \\
-0,588\end{array}$ & $\begin{array}{l}\text { Prob. Culp. } \\
\text { Defensa } \\
\text { Veredicto } \\
\text { Acusación } \\
\text { Umbral } \\
\text { Confianza }\end{array}$ & $\begin{array}{l}4 \\
6 \\
2 \\
5 \\
1 \\
3\end{array}$ & $\begin{array}{r}0,829 \\
-0,790 \\
0,739 \\
0,545 \\
0,037 \\
0,094\end{array}$ & $\begin{array}{r}-0,014 \\
-0,136 \\
-0,396 \\
-0,421 \\
0,773 \\
-0,600\end{array}$ \\
\hline
\end{tabular}


nales los individuos que declaran a todos los acusados culpables o a todos inocentes. Además, la función logarítmica es la que mejor describe el proceso de acumulación de evidencia que determina la adopción de un veredicto (Thomas y. Hogue; 1976). Los cuatro casos utilizados pueden agruparse a los efectos del análisis de varianza, ya que no son significativamente distintos entre sí respecto de la tasa de veredictos a que dan lugar en una muestras de la misma población (Sainz, 1988).

En el análisis de varianza se suponen fijos los efectos de las variables de regla de decisión (mayoría simple o unanimidad), tipo de instrucción (débiles; moderadas y fuertes) y la deliberación, en tanto se expresa en las medidas de la variable dependiente: los efectos de las variables de facultad y de sujeto se suponen, por el contrario, casuales. En el modelo del tèrmino de error se especificaba la variable de sujeto como ligada a la facultad, al tipo de regla y al tipo de instrucción, y las medidas repetidas de la variable dependiente como variable cruzada. Los resultados del análisis arrojan un efecto significativo marginal de la deliberación, con una $F(1,2)=15,89$, $\mathrm{MCe}=3,1405 ; p=0,057$, y un efecto significativo de la interacción entre tipo de instrucción y deliberación, $F(2,4)=51,83, M C \theta=0,6350, p<0,001$. Se obtiene asimismo un efecto significativo, no previsto, de la variable de facultad con una $F(2,90)=5,42$, $\mathrm{MCe}=10,1859, p<0,01$. La tasa media de veredictos de culpabilidad es mayor antes de la deliberación $(0,9193)$ que después de la deliberación $(0,6781)$. La interacción entre tipo de Instrucción y deliberación deriva de obtener unas tasas medias de veredictos previas a la deliberación de 0,8405 , 0,7004 , y 1,2168 para instrucciones fuertes, moderadas y débiles respectivamente, y unas tasas medias de veredictos posteriores a la deliberación de $0,7409,0,5308$, y 0,7226 para instrucciones fuertes, moderadas y débiles, respectivamente (cfr. Fig. 1).

Como se aprecia en la figura 1, la deliberación

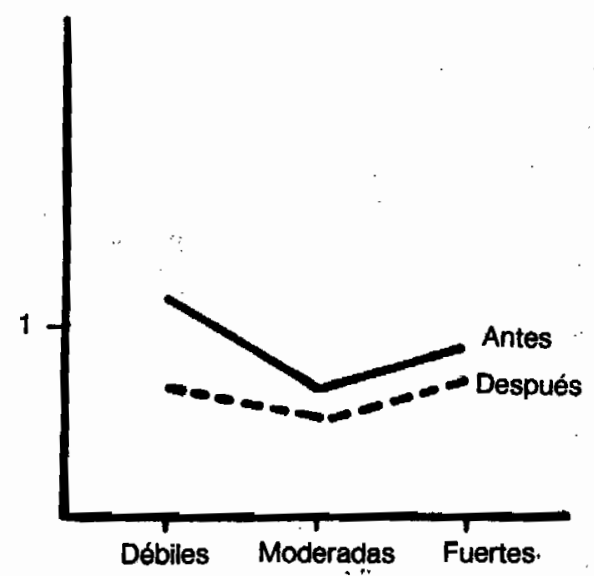

Figura 1. Gráfica de la interacción entre Tipo de instrucción y Deliberación del Experimento 2. tiene el efecto de inclinar el impacto de las instrucciones hacia la no culpabilidad. En tanto las instrucciones fuertes y débiles (una condición de noinstrucciones) tienden a confundir sus efectos tras la deliberación, las instrucciones moderadas se mantienen diferenciadas y favorecen el veredicto de no-culpabilidad.

\section{Discusión}

El umbral de decisión de nuestros sujetos, interpretándolo como criterio normativo en los mismos términos que lo hicieran Nagel, Lamm y Neef (1981), es en la práctica el mismo que encontraran estos autores: un umbral medio de 60 tanto para hombres como para mujeres: El umbral de decisión se medía sin proporcionarles a los sujetos ningún tipo de instrucción acerca del criterio normativo que exige el sistema penal representado por las instrucciones que se proporcionan. Este umbral de decisión expresa que los sujetos prefieren cometer el error de declarar inocente a un culpable antes que el de declarar culpable a un inocente, ponderando como más importante este último tipo de error.

Comoquiera que el test del umbral represerita un criterio genénico de presunción de inocencia, no es claro en qué medida los sujetos variarán su criterio ante un caso concreto. La medida proporcionada por el test de Ostrom, Werner y Saks (1978) muestra que la presunción de inocencia varía según la gravedad del caso confirmando que el hecho de haber sido acusado puede ser entendido por parte de algunos sujetos como un indicio de culpabilidad (Tanford y Penrod, 1984). En términos bayesianos, el hecho de que los sujetos estimen la culpabilidad del acusado según la gravedad de la acusación puede derivar de que basen sus juicios en el conocimiento que tienen de las tasas de veredicto de culpabilidad del sistema judicial para un cierto tipo de acusaciones: Los sujetos aceptan sin un análisis critico la calidad con que trabaja el sistema judicial, supuestamente mayor para los casos más graves. Es dificil distinguir el efecto que esta tasa base tendría en el juicio de los individuos e incluso si es efectivamente utilizada (cfr. Argote y Seabright, 1986), lo que explicaria una tendencia de los sujetos a producir una tasa de veredictos equivalente a la que $s e$ da en el sistema legal (Kerr, Harmon y Graves, 1982):

Que el tiempo de deliberación sea reducido y moderado el tamaño del jurado hace más probable que se parta de una mayoria suficiente para llegar a un veredicto, dependiendo de la condición experimental si es o no crítico el cambio de opinión de uno de sus miembros. Dado que los casos son nivelados, el efecto de la deliberación es consistente: la tasa de veredictos de culpabilidad se reduce en favor del acusado tras la deliberación (cfr. Kerr y MacCoun, 1985). Esta asimetria entre la tasa de veredictos predeliberatoria y postdeliberatoria se ha observado frecuentemente en los estudios sobre la deliberación del jurado (cfr. Kaplan, 1986).

El aumento de confianza tras la deliberación es un 
efecto de la dinámica grupal que permite a sus miembros compartir con la mayoría o con la minoria sus puntos de vista personales (cfr. Stephenson y Davis, 1984). Los análisis de regresión múltiple sobre tasas de veredicto predeliberatorio y postdelibo ratorio muestran las diferentes estrategias de procesamiento seguidas por los sujetos antes y después de la deliberación. En tanto antes de la deliberación su veredicto puede predecirse a partir de la evaluación que les merece la evidencia aportada a favor de la acusación y a favor de la defensa junto con la probabilidad estimada de culpabilidad, tras la deliberación esta última variable deja de intervenir; constituyendo la evidencia del juicio la variable determinante. El hecho de que la probabilidad estimada de culpabilidad deje de ser un predictor tras la deliberación expresa que uno de los efectos de ésta es reducir o eliminar el efecto de una norma subjetiva, en relación con un grupo de referencia, en favor de una norma subjetiva instaurada por el propio jurado a través de la deliberación (cfr. Brookhouse, Guion y Doherty, 1986).

El análisis de varianza, además de poner al descubierto un efecto de la deliberación sobre el veredicto emitido, muestra la forma en que esta variable interactúa con las instrucciones. Dos efectos aquí se combinan: uno de carácter informativo representado por la deliberación, y otro de carácter normativo representado por las instrucciones. El hecho de que sean las instrucciones moderadas las que dan lugar a una tasa menor de veredictos de culpabilidad contradice los resultados de Kerr, Atkin, Stasser; Meek, Holt y Davis (1976) en el sentido de que las instrucciones más estrictas deberian favorecer más al acusado. La razón de esta discrepancia parece en primer término residir en que Kerr y colaboradores (1976) toman como unidad de análisis el grupo, en tanto en esta investigación se ha tomado como unidad el individuo. Sin embargo, esta discrepancia puede estar mostrando distintas estrategias de decisión: los irrdividuos no se exigen una mayor discriminación al dejar en manos del jurado al que perte. necen y antes y después, incluso, al proplo sistema la responsabilidad de filtrar los casos (Grofman, 1981). Desde un punto de vista sistémico, las discriminaciones favorables o desfavorables al acusado son tanto más dificiles de corregir cuanto más avanza el caso por los diferentes nodos del sistema, entre otras causas porque son más dificiles de diagnosticar en base sólo a la información de que se dispone en cada nodo en particular (Black, 1984; Konêcni y Ebbesen, 1982). En ningún caso parece atribulble este cambio de preferencia a una falta de realismo de la simulación. Los comentarios informales de los sujetos acerca de la responsabilidad que habian asumido, sus preguntas acerca del veredicto en su día emitido - ya que se les presentaron los casos como veridicos- $y$, en fin y en general, sus respuestas ante las preguntas abiertas de los cuestionarios avalan el realismo de esta experiencia. Simon (1967), trabajando con jurados reales, ya observó su desinterés por la seritencia a que podía dar lugar su veredicto.
Un efecto indeseable de la facultad muestra algunos de los prejuicios que pueden acompañar a la emisión de un veredicto. Este efecto cabe atribuirlo a un prejuicio experto de los estudiantes. En efecto, los estudiantes, según la cualificación profesional a la que aspiran, se atribuyen este carácter. Ni las instrucciones ni la regla de decisión, como tales, alcanzan significación. Arkes, Dawes y Chnistensen (1986) han constatado empiricamente que aquellos que son o se consideran expertos tienden a utilizar menos una regla de decisión que es útil para la tarea, y que, por tanto, la ejecutan peor.

La polarización de las tasas de veredicto tras la deliberación muestra que ésta introduce cambios que afectan a la evaluación de la evidencia (Kaplan y Miller, 1987), tanto más significativos por cuanto no aparecen relacionados con el prejuicio experto introducido por la variable de facultad. La influencia normativa del jurado sobre cada uno de sus miembros se ve, en cambio, reflejada en el papel de las instrucciones durante la deliberación. Centrado en la evaluación de la evidencia, el jurado parece influir sobre sus miembros a través de un cambio estratégico en el procesamiento de información, que es, tras la deliberación, más analítico. Consistente con nuestras predicciones, la deliberación tiene un efecto sobre el veredicto emitido al suponer un cambio en la estrategia de decisión seguida por los sujetos. Sin embargo, efectos normativos e informativos se hallan confundidos en este experimento. Un tratamiento distinto de la información puede contribuir a distinguir entre ambos efectos.

\section{EXPERIMENTO 3}

Considerando los resultados del segundo experimento no es posible distinguir entre los efectos normativo o informativo del jurado sobre los veredictos emitidos por cada uno de sus miembros, ni tampoco de qué modo afecta el tipo de respuesta que se requiere al uso de estrategias de decisión compensatorias o no compensatorias. Los únicos efectos que se confirman afectan a la deliberación, de carácter informativo cuando se considera su efecto aislado, y de carácter normativo cuando se considera en relación con las instrucciones. La deliberación afecta a la evaluación analítica de la evidencia poniendo en cuestión que el sujeto siga una estrategia de juicio compensatorio cuando, por su cuenta, evalúa la información. Presentando la evidencia. del caso secuencialmente es posible inducir un tratamiento analítico de la información. Este tratamiento afectaria al veredicto reduciendo simultáneamente el papel de la deliberación. En este segundo experimento se trata, precisamente, de distinguir entre ambos efectos, normativo e informativo, de la deliberación y el uso de una estrategia, de elección frente a una estrategia de juicio cuando no se da una evaluación explicita de cada una de las altemativas en relación con la evidencia aportada. 


\section{Método}

\section{Sujetos}

Setenta y dos sujetos efectivos, de edades comprendidas entre los 18 y 44 años, estudiantes del Instituto de Criminología $(n=36)$, y de primer curso de la Escuela de Trabajo Social $(n=36)$ de la Universidad de Valencia participaron voluntariamente en este tercer experimento. Estos sujetos se distribuyeron al azar en 12 grupos de seis personas cada uno, viniendo ya formados a la cita experimental. La relación por sexos era de un 56 por 100 de mujeres, y de un 44 pór 100 de hombres. Cuatro de estos grupos procedian de la sustitución de otros tantos que se descartaron por no acudir todos sus miembros (3) o por no cumplir con los requisitos de la tarea (1). Ninguno de los sujetos que participaron en este experimento había participado en los anteriores.

\section{Diseño}

Se empleó un diseño factorial completo similar al del segundo experimento, 2 (reglas de decisión: mayoria simple o unanimidad) $\times 3$ (tipos de instrucción: débiles, moderadas y fuertes) $\times 2$ (casos de robo: José Linos y Juan Aguilera; véase apéndice I). Al igual que en el segundo experimento, como variable dependiente se adoptó el veredicto predeliberatorio y el veredicto postdeliberatorio del sujeto, es decir, el juicio con el que calificaba la culpabilidad o no culpabilidad del acusado. Sin embargo, en vez de tomar una única medida del veredicto predeliberatorio, se tomaron seis medidas que se corresponden con otras tantas unidades de información (seis proposiciones que especifican la evidencia de cada caso, ordenadas aleatoriamente, tres correspondientes a las pruebas de la acusación y tres correspondientes a las pruebas de la defensa). A la vez que el sujeto emitia su veredicto hacía una estimación de la probabilidad de que el acusado fuera culpable. Las seis estimaciones se consideraban otras tantas medidas repetidas de la segunda variable dependiente de probabilidad de culpabilidad. Se adoptaron las mismas decisiones que en el segundo experimento, por lo que respecta a la toma de las mismas medidas escalares adicionales, y a la asignación de los sujetos a los grupos. No obstante, para reducir el tiempo dedicado a la tarea, aquellas medidas escalares se tomaron únicamente antes de la deliberación. Todas las variables experimentales se manipularon de la misma forma. La única diferencia, razón de que se lleve a cabo este experimento, se refiere a la presentación secuencial de la información.

\section{Material de estímulo y procedimiento}

El material de estímulo era, por completo, idéntico al presentado en el segundo experimerito, excepto, por lo que se refiere a la presentación de dos únicos casos, a la presentación secuencial de la información especifica de cada caso, a la toma de seis medidas del veredicto predeliberatorio y a la toma de seis estimadas predeliberatorias de probabilidad de culpabilidad. El procedimiento era idéntico al segui: do en el segundo experimento, excepto en que se requeria de los sujetos que emitieran su veredicto y que estimaran la probabilidad de culpabilidad del acusado tras cada unidad de información, y en que sólo rellenaban el cuestionario predeliberatorio.

\section{Resultados}

El comportamiento de los sujetos en los tests de Nagel, Lamm y Neef (1981) y de Ostrom, Werner y Saks (1978) es sensiblemente idéntico al del segundo expenimento. El umbral de decisión alcanza una puntuación media de 57,95; la disposición previa varía, dependiendo de la gravedad del delito, en el mismo rango. Las puntuaciones medias en las distintas escalas del cuestionario predeliberatorio no son significativamente distintas entre ambos casos (en confianza, 57,04 vs 57,12 ; en probabilidad de culpabilidad, 47,41 vs 43,12 ; en pruebas a favor de la acusación, 32,50 vs 34,64; en pruebas a favor de la defensa, 35,89 vs 39, para los casos José Linos y Juan Aguilera, respectivamente), confirmando que son equilibrados por lo que respecta a estas escalas de medida. Sin embargo, al contrario que en el segundo experimento, los sujetos producen tasas de veredictos finales predeliberatorias no equilibradas. La tasa de veredicto predeliberatoria final es de tan sólo un 12,3 por 100 de veredictos de culpabilidad. La tasa de veredicto postdeliberatoria es aún menor, del orden de un 9,2 por 100 de veredictos de culpabilidad.

A fin de ejecutar los sucesivos análisis sobre distribuciones normalizadas de los datos originales, se calcularon los antilogaritmos de las puntuaciones medias obtenidas en las distintas escalas para los dos casos, y los antiloganitmos de la proporción de veredictos de no-culpabilidad y de probabilidad de culpabilidad sobre estos dos casos, en las seis medidas respectivas de estas variables, proporcionadas tras cada ítem de información.

Sometiendo las puntuaciones escalares predeliberatorias obtenidas a un anélisis factorial de componentes principales, el 59,24 por 100 de la vanianza puede explicarse en términos de tres factores; en el espacio factorial, el factor de evidencia en el que ponderan positivamente las escalas que califican la fuerza de las pruebas de la acusación y de la defensa, explica el 37,91 por 100 de la varianza; el segundo factor de no culpabilidad, en el que ponderan positivamente la tasa de veredictos y negativamente las escalas que califican la confianza en la decisión adoptada y la probabilidad de que el acusado sea culpable explica el 31,99 por 100 de la varianza; y el tercer factor de consistencia, en el que ponderan positivamente el umbral de decisión y negativamente la probabilidad de culpabilidad, explica el 30,10 


\section{CUADRO 2}

Pautas y cartas factoriales rotadas y predellberatorias en el análisis factorial sobre tasas de veredicto y puntuaciones escalares finales del tercer experimento

\begin{tabular}{|c|c|c|c|c|c|}
\hline & Pauta & Var. & Factor 1. & Factor 2 & Factor 3 \\
\hline $\begin{array}{l}\text { Acusación } \\
\text { Defensa } \\
\text { Veredicto } \\
\text { Confianza } \\
\text { Umbral } \\
\text { Prob. Culp. }\end{array}$ & & $\begin{array}{l}5 \\
6 \\
2 \\
3 \\
1 \\
4\end{array}$ & $\begin{array}{l}0,824 \\
0,735 \\
0,155 \\
0,194 \\
0,013 \\
0,061\end{array}$ & $\begin{array}{r}0,041 \\
-0,108 \\
0,757 \\
-0,605 \\
-0,194 \\
-0,428\end{array}$ & $\begin{array}{r}0,175 \\
-0,213 \\
-0,157 \\
-0,083 \\
0,833 \\
-0,543\end{array}$ \\
\hline
\end{tabular}

\section{CUADRO 3}

Pautas y cargas factorlales rotadas predeliberatorias en el análisis factorial sobre tasas de veredicto y puntuaciones escalares parciales, por ítem de información, del tercer experimento

\begin{tabular}{lrrrr}
\hline \multicolumn{1}{c}{ Pauta } & Var. & Factor 1 & Factor 2 & Factor 3 \\
\hline Acusación parcial 3 & 4 & 0,975 & 0,130 & 0,129 \\
Acusación parcial 5 & 10 & 0,970 & 0,084 & 0,127 \\
Defensa parcial 4 & 8 & 0,969 & 0,112 & 0,142 \\
Defensa parcial 6 & 12 & 0,964 & 0,081 & 0,137 \\
Acusación parcial 1 & 2 & 0,942 & 0,182 & 0,086 \\
Defensa parcial 2 & 4 & 0,937 & 0,155 & 0,124 \\
Tasa de veredicto 1 & 1 & 0,089 & 0,868 & $-0,124$ \\
Tasa de veredicto 2 & 3 & 0,073 & 0,797 & 0,197 \\
Tasa de veredicto 3 & 5 & 0,160 & 0,783 & 0,428 \\
Tasa de veredicto 4 & 7 & 0,197 & 0,676 & 0,345 \\
Tasa de veredicto 6 & 11 & 0,142 & 0,160 & 0,913 \\
Tasa de veredicto 5 & 9 & 0,182 & 0,238 & 0,842 \\
\hline
\end{tabular}

por 100 de la varianza (cfr. cuadro 2). Este último factor es de una importancia especial: siempre que la probabilidad estimada de culpabilidad es menor que el umbral de decisión puede juzgarse que el sujeto es consistente al declarar no-culpable al acusado. Determinando esta correspondencia obtenemos que el 80,8 por 100 de los sujetos se comportaban de forma consistente. Además, se constata que hay tendencia a que los veredictos de culpabilidad se den con menos confianza que los veredictos de no-culpabilidad y en proporción inversa a la estimada de probabilidad de culpabilidad $(r=-0,21$. p $<0,01)$.

Sometiendo a análisis factorial las tasas de veredicto y las puntuaciones escalares de probabilidad de culpabilidad, que se obtienen tras la presentación de cada ítem de información, obtenemos una configuración factorial descriptiva de cómo evalúa el sujeto la evidencia. En efecto, tres factores bastan para explicar el 85,76 por 100 de la varianza en el espacio de los datos. En el primer factor de noculpabilidad ponderan las distintas estimadas de probabilidad de culpabilidad proporcionadas por los sujetos tras cada veredicto. Este factor explica el 63,28 por 100 de la varianza en el espacio factorial. El segundo factor, en el que ponderan las tasas de veredicto parciales, es un factor de veredicto que describe el proceso de acumulación de evidencia. Este factor explica el 25,04 por 100 de la varianza. El-tercer factor, por último, en el que ponderan sólo los items de información finales es un factor de veredicto que describe la adopción de una decisión definitiva. Este factor explica el 11,68 por 100 de la varianza (cfr. cuadro 3 ).

Un análisis de regresión múltiple, llevado a cabo para detectar el mejor conjunto de predictores del veredicto predeliberatorio a partir de las escalas del cuestionario previo, no nos proporciona ningún subconjunto de variables que sea un predictor significativo del veredicto final. Sin embargo, llevando a cabo un análisis de regresión múltiple adoptando como variables de criterio las tasas de veredicto y las probabilidades de culpabilidad obtenidas tras la presentación de cada ítem de información, obtenemos un modelo predictor del veredicto final. En efecto, los tres veredictos que siguen a la presentación de pruebas a favor de la acusación es el conjunto de variables que mejor predice el veredicto final. Estos predictores explican el 61,51 por 100 de la varianza, con una $F(3,68)=36,23$, $p<0,001$.

Para una primera aproximación al análisis de la varianza se llevó a cabo un análisis de varianza mix- 
to. sobre las tasas de veredicto proporcionadas antes y después de la deliberación con el mismo tratamiento que el dado al segundo experimento. En este análisis no se observó ningún efecto significativo de las variables experimentales manipuladas. Se llevó a cabo, entonces, un análisis de varianza mixto con casillas de igual tamaño sobre las seis medidas repetidas de las dos variables dependientes de tasa de veredictos y probabilidad de culpabilidad; en el que se suponen fijos los efectos de las variables de regla de decisión (mayorla simple o unanimidad), tipo de instrucción (débiles, moderadas y fuertes), nivel de información (de una a seis proposiciones que presentan evidencia a favor de la acusación y a favor de la defensa) y tipo de medida (tasa de veredicto y probabilidad estimada de culpabilidad) y casuales los efectos de las variables de facultad y sujetos. En análisis posteriores se distingue el tipo de evidencia (carácter de las proposiciones de presentar evidencia a favor de la acusación o de la defensa) al efectuar el análisis por separado sobre cada medida dependiente. Los efectos del tipo de evidencia y los efectos de la emisión de un veredicto o de la estimación de una probabilidad de culpabilidad ante una presentación secuencial de la información se suponen fijos. En el modelo del término de error se especificaba la variable de sujeto como ligada a la facultad, al tipo de regla y al tipo de instrucción, y las medidas repetidas de las variables dependientes como variables cruzadas. Posteriores análisis se organizan a este respecto de la misma forma.

Los resultados del análisis arrojan un efecto significativo de la variable de facultad con una $F(1,60)=$ $=12,99, \mathrm{MC} e=5,81069, p<0,001$ y de sus interacciones con las variables tipo de instrucción con una $F(2,60)=8,85, M C e=5,81069, p<0,001$, por una parte, y tipo de medida, por otra, con una $F(1,60)=$ $=4,00, \mathrm{MCe}=2,84352, \mathrm{p}<0,05$. Resulta ser también significativa la variable de nivel de información con una $F(5,5)=6,27, M C e=0,30388$, $p<0,05$, un efecto que deriva de la presentación secuencial de la información. La variable tipo de medida resulta ser marginalmente significativa con una $F(1,1)=$ $=117,13, M C_{\theta}=11,38370, p=0,0587$. El interés de este efecto y el del que se refiere a la interacción en que participa es marginal, dado que las escalas de medida son diferentes en ambas variables dependientes (cfr. Parducci y Wedell, 1986; Parducci, 1988). Ni la variable de instrucción con una $F(2,2)=$ $=1,22, \mathrm{MCe}=51,43686, \mathrm{p}>0,05$, ni la de regla con una $F(1,1)=17,44, \mathrm{MCe}=0,57089, p=0,1496$ resultan ser significativas. En este análisis, sin embargo, se han combinado los efectos de cada variable dependiente, por lo que resulta útil aclarar, con nuevos análisis, el distinto comportamiento de las tasas de veredicto y las probabilidades estimadas de culpabilidad a medida que se acumula más información sobre los respectivos casos.

Un análisis de varianza llevado a cabo sobre las tasas de veredicto muestra que la facultad no tiene un efecto significativo con una $F(1,60)=2,33, \mathrm{MCe}=$ $=5,81069, p>0,05$. Si es significativo el efecto del tipo de evidencia, es decir, el efecto que deriva de si es a favor de la acusación o a favor de la defensa, con una $F(1,1),=669,22, \mathrm{MCe}=0,0080316$, $p<0,05$. La variable regla de decisión, que no resulta ser significativa, se comporta, sin embargo, de la forma esperada, a saber, una tasa menor de veredictos de no-culpabilidad con la regla de mayona simple $(0,56916)$ que con la regla de unanimidad $(0,86182)$ de acuerdo con la tendencia prevalente en el grupo. Ningún otro efecto es significativo.

En el análisis de varianza llevado a cabo sobre probabilidades estimadas de culpabilidad, la variable de facultad resulta ser significativa con una $F(1,60)=$ $=27,93, \mathrm{MCe}=2,6046, p<0,001$. La interacción entre tipo de instrucción y tipo de evidencia, con una $F(2,2)=33,75, M C e=0,0066784, p<0,05$, también resulta ser significativa, mostrando que las instrucciones afectan a la evaluación de la evidencia, a la probabilidad de culpabilidad que se estima tras cada ítem de información. Las instrucciones débiles y fuertes dan lugar a estimaciones de probabilidad de culpabilidad más altas que las instrucciones moderadas tanto cuando se presentan pruebas a favor de la acusación $(3,66938$ y 3,84267 , respectivamente) como cuando se presentan a favor de la defensa $(3,55607$ y 3,80119 , respectivamente). Con instrucciones moderadas esta relación se invierte en parte $(2,14317$ vs 2,18790 , para la acusación y la defensa respectivamente) (cfr. Fig. 2).

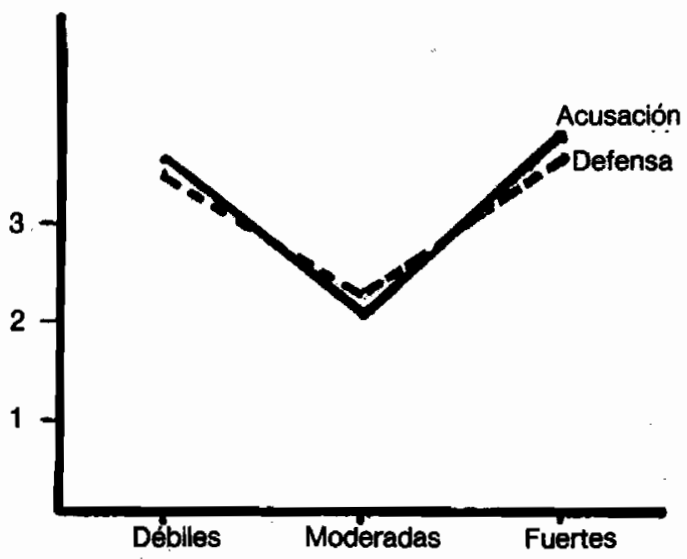

Figura 2. Gráfica de la interacción entre Tipo de instrucción y Probabilidad estimada de culpabilidad del Experimento 3.

A fin de comparar los resultados obtenidos en el segundo y tercer experimentos, un último análisis de varianza resulta de interés. Sobre las tasas de veredicto proporcionadas antes y después de la deliberación, se llevó a cabo un análisis de varianza para probar la igualdad de las medias entre ambos tratamientos. Ningún efecto resulta ser significativo excepto por to que se refiere al tipo de experimento 
como variable en sentido propio, que se introduce al efectuar la comparación, con una $F(1,178)=3,79$, $\mathrm{MCe}=2,57474, \mathrm{p}<0,05$. Ambos tratamientos se comportan globalmente de distinta forma, pero este distinto tratamiento no se refleja sistemáticamente en alguna de las variables experimentales manipuladas.

\section{Discusión}

De acuerdo con nuestra hipótesis bésica, la manipulación de la forma en que se presenta la información referente al caso tiene un efecto dramático en la tasa de veredictos al alterar las estrategias de procesamiento de la información empleadas por los sujetos. Dado que el umbral de decisión es idéntico en los dos últimos experimentos, el hecho de que la tasa de veredictos predeliberatoria sea baja desmiente la hipótesis de que los sujetos se atienen estrictamente, a la hora de decidir su veredicto, a su umbral y disposición prevla. La estrategia adoptada para evaluar la evidencia juega un papel obviamente crítico. El efecto de aquellas variables aparece asociado, en el análisis factorial realizado sobre tasas de veredicto y probabilidades estimadas de culpabilidad, al papel que juega la evidencla.

El análisis de regresión llevado a cabo, adoptando como variables predictoras del veredicto final las tasas de veredicto predeliberatorias y las probabilidades estimadas de culpabilidad, indica que las tasas de veredicto referidas a proposiciones incriminatorias constituyen las variabies que mejor predicen el veredicto final. Este efecto es tarto más fiable por cuanto los sujetos no conocen por anticipado a qué categoría de veredicto pertenece cada proposición y los casos que se presentan dan lugar a tasas de veredicto equivalentes. Este proceso de acumulación de evidencia (cfr. Thomas y Hogue, 1976) le permite al sujeto adoptar una decisión basándose en la calidad de la información incriminatoria aportada en el juicio. De acuerdo con el criterio de presunción de inocencia es la parte acusadora la que tiene que demostrar la culpabilidad del acusado. La calidad de la evidencia aportada por la defensa, en si misma considerada, no condiciona su veredicto. Las pruebas de la defensa son utilizadas por los sujetos para compensar y moderar el impacto de la evidencla incriminatoria.

El análisis de varianza llevado a cabo sobre las tasas de veredicto muestra que la variable de facultad no tiene un efecto significativo. Sobre el veredicto influye el tipo de evidencia, de si es favorable a la acusación o a la defensa. No se da por tanto un efecto de prejuicio experto en relación con el veredicto. Éste se manifiesta, en cambio, en la evaluación que el sujeto hace del caso a través de las probabilidades estimadas de culpabilidad. La interacción de esta vaniable con las instrucciones muestra que este prejuicio experto tiene sólo un efecto normativo y no influye sobre la evaluación de la evidencia. Las instrucciones fuertes y débiles producen estimadas de probabilidad de culpabilidad más al- tas que las instrucciones moderadas al igual que ocurre con la tasa de veredictos en el segundo experimento.

Los hallazgos sin duda más relevantes de este experimento se refieren a la ausencia de un efecto debido a la deliberación junto con la baja tasa de veredictos de culpabilidad obtenidos. La única variable crítica para estos hallazgos es la presentación secuencial de la información: el tipo de respuesta que se requiere induce en los sujetos una estrategia compensatoria y una evaluación analítica de la información aportada. Esos resultados explican la naturaleza informativa del efecto de la deliberación del experimento anterior, que se ve reducido o eliminado por un cambio de estrategia del sujeto, a partir de la presentación secuencial de la evidencia. El efecto de esta manipulación experimental es claro en la significación que alcanza la variable de nivel de información y en el contraste significativo de este experimento con el anterior. En relación con esta misma hipótesis, la regla de decisión presenta una fuerte tendencia a la significación, lo que explica su influencia, de carácter informativo, en el proceso de decisión. El que la regla de decisión no resulte ser significativa en otro contexto puede depender de la estrategia empleada por el sujeto para evaluar la Información que determina su veredicto.

\section{Discusión general}

Los sujetos evalúan la evidencia, respecto de si es suflclente o no para dar un veredicto de culpabilidad, en términos de su valor incriminatorio. El proceso de evaluación consiste, cuando se sigue una estrategia de juicio analítica y compensatoria, en asignar un valor a cada proposición, según su valor incriminatorio, de acuerdo con una función de acumulación hasta un nivel de saturación de evidencia o nivel umbral. Los indicios se combinan de forma multiplicativa según este proceso de acumulación de evidencia que concluye en una estimada final de probabilidad de culpabilidad. Los sujetos, miembros de un jurado, siguen una estrategia compensatoria para juzgar la evidencia aportada por la defensa. Este juicio de probabilidad estimada de culpabilidad se contrasta, entonces, con el nivel umbral. Si la probabilidad estimada es inferior, el sujeto decide la no culpabilidad, decidiendo la culpabilidad en el caso contrario.

La influencia de la deliberación sobre el veredicto obtenida en el segundo experimento es, a la luz de los resultados del tercer experimento, de naturaleza informativa: El procesamiento analítico de la información a que obliga la presentación secuencial de la evidencia y el hecho de que se requiera una evaluación explicita de ésta en relación con el veredicto da lugar a una caída en la tasa de veredictos de culpabilidad antes de la deliberación. Esta tasa tan baja de veredictos de culpabilidad contrasta dramáticamente con la obtenida en el segundo experimento utilizando los mismos casos. Billings y Scherer (1988) han mostrado que el tipo de respuesta que 
se requiere condiciona la estrategia que el sujeto emplea para elaborar la información.

De acuerdo con Billings y Scherer (1988), ta presentación secuencial de la información obligaría a los sujetos a una comparación interdimensional entre las alternativas, es decir, a enjuiciar la evidencia explícitamente, en tanto que la información global del caso, tal como se proporciona en el segundo experimento, llevanía a una elección entre las alternativas sin enjuiciar la información. La presentación secuencial lleva a utllizar una estrategia compensatoria: los sujetos sienten que se les pide que realicen un juicio y emplean entonces una estrategia más elaborada. Cuando, por el contrario, se presenta simultáneamente toda la información del caso, los sujetos entienden que se les pide que realicen una elección y adoptan una estrategia no compensatoria que reduce la carga de información, como, por ejemplo, una estrategia de eliminación por aspectos. No es posible decidir a priori qué estrategia dará lugar a resultados más precisos. En una tarea de decisión compleja contando con pocos recursos, una estrategia de eliminación por aspectos puede ser más útil (Paquette y Kida, 1988).

Nuestros resultados muestran que son las diferentes estrategias empleadas en la elaboración de la información las responsables de que se produzca una tasa diferencial de veredictos. Esta interpretación se ve avalada por el hecho de que los casos se basen en rigor en pruebas circunstanciales y no se proporcionen pruebas que establezcan inequivocamente la identidad de los acusados. Esta caída de la tasa de veredictos, que sigue a un cambio de estrategia, no puede explicarse como un efecto del prejuicio experto: el análisis de varianza muestra que éste no influye en las tasas de veredicto sino en los componentes más nomativos del proceso. Por otra parte, este prejuicio experto también se daba en el segundo experimento y no se observaba una tasa tan baja de veredictos de culpabilidad con los mismos casos.

\section{Referencias}

Arkes, H. R.; Dawes, R. M., y Christensen, C. (1986): Factors influencing the use of a decision rule in a probabilistic task, Organizational behavior and human decision processes, 37, 93-110.

Argote, L., y Seabright, M. A. (1986): Individual versus group use of base-rate and individuating information, Organizational behavior and human decision processes, $38,65-75$.

Bettman, J. R. (1982): A functional analysis of the role of overall evaluation of altematives in choice processes, Advances in Consumer Research, 9, 87-93.

Billings, A.S., y Scherer, L. L. (1988): The effects of response mode and importance on decision-making strategies: Judgment versus choice, Organizational behavior and human decision processes, 41, 1-19.

Black, D. (1984): Toward a general theory of social control, 2 vol., New York, Academic Press.

Brookhouse, K. J.; Guion, R. M., y Doherty, M. E. (1986): Social desirability response blas as one source of the discrepancy between subjective weights and regression weights, Organizational behavior and human decision processes, 37, 316-328.

Christensen-Szalanski, J. J. J. (1986): Improving the practlcal utility of judgment research. En B. Brehmer; H. Jungermann; P. Lowens, y G. Sevon (eds.): New directions in research on decision making, New York, North Holland.

Davis, J. H.; Kerr, N. L.; Atkin, R. S.; Holt, R., y Meek, D. (1975): The decision processes of 6- and 12-person mock juries assigned unanimous and two-thirds majority rules, Journal of Personality and Social Psychology, 32. $1,1-14$

Einhorn, H. J.; Kleinmuntz, D. N., y Kleinmuntz, B. (1979): Linear regression and process-training models of judgments, Psychological Review, 86, 465-485.

Feeley, M. M. (1983): Court reform on trial. Why simple solutions fail, New York, Basis Books.

Grofman, B. (1981): Mathematical models of juror and jury decision-making. The state of the art. En B.D. Sales (ed.): The trial process, 305-347, New York, Plenum Press.

Kaplan, M.F. (1986): Social psychological assumptions and dimensions in judicial systems, Conferencia en el International Congress on judicial criminology, psychology and sociology, Allcante, 28-31 de octubre.

Kaplan, M. F. y Miller, C. E. (1987): Group decision making and normative versus informational influence: Effects of type of issue and assigned decision rule, Journal of Personality and Social Psychology, 53, 2, 306-313.

Kerr, N.L. (1985): On the cutting edge of jury research, Contemporary Psychology, 30, 1, 5-7.

Kerr, N. L.; Atkin, R. S.; Stasser, G.; Meek, D.; Holt, R.W., y Davis, J. H. (1976): Guilt beyond a reasonable doubt: Effects of concept definition and assigned decision rule on the judgments of mock juries, Journal of Personality and Soclal Psychology, 34, 2, 282-294.

Kerr, N. L.; Harmon, D. L., y Graves, J. K. (1982): Independence of multiple veredicts by jurors, Journal of Applied Social Psychology, 12, 1, 12-29.

Kerr, N. L., y MacCoun, R. J. (1985): The effects of jury size and polling method on the process and product of jury deliberations, Journal of Personality and Social Psychology, 48, 2, 349-363.

Konêcni, V.J., y Ebbesen, E. B. (1982): Social psychology and the law: The choice of research problems, settings, and methodology. En V.J. Konécni, y E. B. Ebbesen: The criminal justice system: A social psychological approach, San Francisco, Freeman.

Nagel, S. S.; Lamm, D., y Neef, M. (1981): Decision theory and juror decision making. En B. D. Sales (ed.): The trial process, 353-384, New York, Plenum Press.

Ostrom, T. M.; Weerner, L., y Saks, M. J. (1978): An integration theory analysis of juror's presumptions of guilt of innocence, Journal of Personality and Social Psychology, 36, 4, 436-450.

Payne, J.W. (1982): Contingent decision behavior, Psychological Bulletin, 92, 382-402.

Paquette y Kida (1988): The effect of decision strategy and task complexity on decision pertormance, Organizatlo$\mathrm{nal}$ behavior and human decision processes, 41, 128142.

Pennington, N., y Hastie, R. (1986): Evidence evaluation in complex decision making, Journal of Personality and Soclal Psychology, 51, 2, 242-258. (Traducido on este volumen.)

Sainz, E. (1988): La simulación de juicios con jurados, Cuadernos de Politica Crim/nal, 35.

Scheflin, A. W., y VanKÿke, J. (1980): Jury nullification: The contours of a controversy, Law and contemporary problems, 43, 4, 51-115. 
Simon, R. J. (1967): The jury and the defense of insanity, Boston, Little Brown.

Stephenson, G. M., y Davis, J. H. (1984): Progress In applled social psychology, 2, New York, John Wliey and sons.

Tanford, S., y Penrod, S. (1984): Social influence model: A formal integration of research on majority and minority influence processes, Psychologlcal Bulletin, 95, 2, 189225.

Tanford, S., y Penrod, S. (1986): Jury deliberations: Discussion content and influence processes in jury decision making, Journal of Applied Social Psychology, 16, 4, 322-347. (Traducido en este volumen.)

Thomas, E. A. C., y Hogue, A. (1976): Apparent weight of evidence; decision criteria, and confidence ratings in juror decision making. Psychologlcal Revlew, 83, 6, 442465.

Tversky, A. (1977): Features of similarity, Psychological Review, 84, 327-352.

Wallsten, T. S., y Budescu, D. V. (1983): Encoding subjective probabilities: A psychological and psychometric review, Management Science, 29, 151-173.

\section{APÉNDICE I}

\section{Instrucciones generales respecto del caso $\theta$ instrucclones débiles}

El cargo que se hace al acusado es de robo. El Código Penal lo define asi:

«Son reos del delito de robo los que, con ánimo de lucrarse, se apoderan de las cosas muebles ajenas con violencia o intimidación en las personas o empleando fuerza en las cosas."

\section{Instrucciones moderadas}

Tanto la ACUSACIÓN como la DEFENSA deben convencerle de que las pruebas y testimonios que aportan les favorecen. La parte de la acusación no tlene que convencerle de que el acusado es culpable sin abrigar la más minima duda, ni tampoco la parte de la defensa debe convencerle de que el acusado es inocente sin abrigar la más minima duda. Su responsabilidad es determinar si la evidencia aportada favorece a la parte acusadora o a la defensora, y votar de acuerdo con ello.

Recuerde que ninguna de las partes necesita probar que los hechos son absolutamente verdaderos. Por supuesto que puede dudarse de un hecho evidente, pero dudar no es siempre idéntico a dudar de una forma razonable. Una duda razonable no es ni caprichosa ni trivial, es seria y se funda en una presunción de culpabilidad o de inocencia del acusado sólo en relación con el delito de que se le acusa.

Debe basar sus dudes sobre la evidencia que se le presenta del caso y no sobre un prejuicio o sobre algo no explicitamente presentado. Debe pensar en las razones que justifican su decisión. Sin embargo, no necesita estar absolutamente seguro de que el acusado es culpable 0 inocente para emitir su juicio.

\section{Instrucctones fuertes}

De acuerdo con la ley, el acusado es inocente hasta que no se demuestre lo contrario. Para que usted cambie su presunción de inocencia, la ACUSACIÓN tiene que convencerle para que usted tenga certeza moral, con pruebas positivas y fehacientes de que el acusado es culpable. Es decir, para que pueda dar un veredicto de culpabilidad, usted debe estar seguro y convencido de que el acusado. es culpable. Si no tiene seguridad y convicción, debe usted declararlo no-culpable. Al revisar las pruebas del caso, puede usted formarse diversas representaciones acerca de lo que puede haber ocurrido. Una de estas representaciones es, por supuesto, la de que el acusado es culpable del cargo del que se le acusa. Pero puede que usted sea capaz de explicar los hechos de otro modo. Si cree que los hechos del caso son compatibles con cualquier otra representación además de aquella que supone que el acusado es culpable, entonces usted tiene una duda razonable respecto de la culpabilidad del acusado. En este caso, debe declararlo no-culpable. Si usted está convencido de que no tiene una duda razonable, debe declararle culpable.

En resumen, para declarar culpable al acusado, debe creer más allá de cualquier duda razonable que el acusado es culpable del delito de robo tal y como se ha definido.

\section{APÉNDICE ||}

\section{Caso: Julio de Castro}

El 19 de febrero de 1980, a las 8,45 de la tarde, una gasolinera fue robada por un hombre joven. El ladrón amenazó por la espalda al empleado, por lo que éste apenas pudo ver que llevaba unas botas del ejército y que escapaba en un seat 124 de color blanco. Julio de Castro fue detenido a las 10,30 de la noche en su apartamento, doce edificios más allá de la gasolinera, cuando la policía encontró un seat 124 blanco que se correspondía con la descripción dada por el empleado de la gasolinera. El registro de coches matriculados reveló que el coche pertenecía a Julio de Castro, que vivia en el edificio de apartamentos que estaba frente ai coche. Un registro del apartamento de Julio de Castro descubrió la existencia de unas botas milltares y 33.000 pésetas en un armario-ropero.

Julio de Castro testifico que había acabado recientemente el servicio militar y que por este motivo tenía botas milltares. También dijo que estaba en casa solo cuando el robo ocurrió. El portero de la finca testifico que habia visto entrar a De Castro en el apartamento esa tarde, pero que sólo podía conjeturar la hora en que sucedió salrededor de las 9». Julio de Castro dijo que habia ganado el dinero en las quinielas.

\section{Caso: Juan Aguilera}

Juan Aguilera fue detenido en su vivienda después que la policla averiguó a quién correspondia la matricula de un vehiculo usado en el robo de unos grandes almacenes; la matrícula correspondía al coche de Aguilera. En posesión de Aguilera se encontró una pequeña bolsa de las que se emplean en esos grandes alimacenes para despachar la mercancia, con dinero suelto. Debido a que el ladrón llevaba una máscara, el empleado fue incapaz de identificar a Aguilera, pero el empleado testifico que el ladrón tenía un tatuaje en su mano derecha al igual que Aguilera.

Carlos Pardo, propietario de un bar, testificó que el día en cuestión él vio en su establecimiento cómo Aguilera daba las llaves de su coche a un hombre llamado *Eco*. «Eco» dejó el establecimiento y más tarde se fue Aguilera. "Eco" y Aguilera volvieron por separado pasadas unas horas esa misma tarde, y "Eco» devolvió las llaves a Aguilera. Un artista local testificó que en una ciudad portuaria habla cientos de personas con tatuajes en la mano. Aguilera testifico que él empleaba la pequeña bolsa de los grandes almacenes para ahorrar dinero. 


\section{Caso: Gulliermo Lizcano}

A primeras horas de la tarde del 27 de marzo de 1980, un bar-restaurante fue robado a punta de cuchillo por un joven enmascarado. Mientras cogia el dinero a través de la ventanilla que daba a la calle, el ladrón se cortó la muñeca izquierda con el marco de la ventana dejando numerosas manchas de sangre en el mostrador. El análisis de la pollcía demostró que la sangre era del tipo $A B$ negativo, un tipo poco frecuente. Una hora después del robo la policia detuvo a Guillermo Lizcano en la sala de emergencia de un hospital donde se le estaba tratando de una herida de cuchillo en la muñeca izquierda. Tras registrar a Lizcano, la policía encontró una navaja automática y 30.000 pesetas en billetes de mil y de cien pesetas. Las pruebas de laboratorio demostraron que la sangre de Lizcano era del tipo $A B$ negativo. Frente a esta evidencia, Lizcano se confesó autor del robo, pero más tarde ante el juez negó haber sido el autor del robo.

El doctor Francisco Giménez, psiquiatra, testificó que Lizcano tenía una personalidad compulsivo-obsesiva que podia llevarle a confesar cualquier cosa. Los antecedentes policiales mostraban que Lizcano se habia confesado autor de un crimen, dos años antes, que no habia cometido. Giménez testificó además que Lizcano se había automutilado más de una vez en los dos últimos años. Estas automutilaciones habían afectado a una o ambas manos, muñecas $\mathrm{O}$ antebrazos. El doctor también señaló que aunque el grupo $A B$ negativo es un tipo de sangre poco frecuente, se pueden encontrar varios millares de personas en la región con el tipo AB negativo.

\section{Caso: José Linos}

A las 8,20 de la tarde del 18 de abril de 1980, Antonia Rais, de 72 años de edad, fue robada a punta de navaja por un hombre joven enfrente de su apartamento. Se llevó su cartera con 4.000 pesetas, un reloj y su anillo de casada. El acusado, José Linos, fue detenido al día siguiente tras haber empeñado el anillo robado. Cuando fue detenido llevaba una navaja automática. La señora Rais no pudo identificar al joven, pero dijo que el acusado se parecía mucho a su asaltante. Un peatón que había visto a un joven correr cerca de la escena del dellto, dijo que el acusado tenia la misma apariencia en general, altura y aspecto corporal que la persona a la que él había visto corriendo.

Linos testificó que habia comprado el anillo a un extraño, y que pensó que podia ganar dinero empeñándolo. Él testificó que estaba en casa, viendo la televisión a la hora en que se cometió el delito, y describió con precisión el contenido del programa emitido a esa hora. Más tarde, la parte acusadora estableció que el programa era la segunda vez que se emitía con idéntico contenido, el acusado no tenia arrestos previos y su jefe dijo que era un buen empleado. 